\title{
CONTROL DE CALIDAD \\ DE LOS FITOFÁRMACOS: \\ Ecuador uso y comercio de plantas medicinales. Si- tuación actual y aspectos importantes para su con- servación
}

Dr. Marco Dehesa G. *

$\mathrm{L}$

os fitofármacos constituyen una terapia que cuando se realiza sobre bases científicas y técnicas, se convierten en una alternativa necesaria en nuestro país, por muchas razones. Estos medicamentos deben tener calidad total, para que la población tenga acceso a un tratamiento medicamentoso, seguro, eficaz y sobre todo, económico.

Para que esto se cumpla lo primero que tiene que entenderse es que los farmacéuticos somos los profesionales que podemos garantizar ese tipo de tratamiento y liderar a través de formar todo el proceso de obtención de un fitomedicamento, por supuesto formando parte de un equipo multidisciplinario, necesario para acometer el proceso cuyo producto final es un fitofármaco.

En el ámbito internacional existen muchas referencias acerca de la importancia y necesidad de obtención y utilización de las plantas medicinales como medicamentos. En este aspecto, es desde antes de la existencia misma del hombre cuando los animales acudieron a las plantas para curar sus afecciones y dolencias. Entonces las comunidades de homínidos y primitivos indígenas acumularon; durante milenios, conocimientos de las plantas, que hoy al ser revelados, sorprenden a la comunidad científica. (1).

En muchos países de la Comunidad Económica Europea se comercializan e investigan los fitofármacos, y además, existe un programa muy importante que nos da un espacio, ya que fomen-

*Director Maestría en Tecnologías para el Aprovechamiento de los Recursos Naturales no Tradicional

Proyecto VIS-UPS Sede Quito, Coordinador de la Red RIPROFITO Ecuador. 
ta la investigación y desarrollo en el campo de las plantas medicinales, que es el CYTED (Ciencia y Tecnología para el DesarroIlo de Iberoamérica) con el Sub programa X Química Fina Farmacéutica y dentro de este, diferentes redes como RIPROFITO (Red de Productos Fitofarmacéuticos) en la que el Ecuador está integrado con un grupo de Laboratorios Fitofarmacéuticos, y ha empezado a trabajar con seriedad en el tema.

Había mencionado la necesidad de contar con fitomedicamentos de calidad y en este sentido, la OMS ha dado pautas para el control de medicamentos herbarios (2). Existen otras normativas internacionales como son de la ESCOP y TRAMIL. En muchos países de Latinoamérica ya se han logrado legislaciones que garantizan el cumplimiento de las normas internacionales para el Registro y la Comercialización de los fitofármacos. En el Ecuador venimos trabajando desde 1995 en una normativa que garantice el marco legal para la utilización de los productos naturales de uso medicinal. Afortunadamente, se ha logrado oficializarla a través del Ministerio de Salud Pública (Registro Oficial 186 del 7 de mayo de 1999). Me refiero a esto, porque en mi criterio, de allí tenemos que partir para lograr el objetivo final del que estamos hablando: el fitomedicamento accesible al paciente.

En el capítulo VI de esta Reglamentación para el registro y control de productos naturales de uso medicinal, se plantea lo concerniente al control de calidad de estos productos y se establece que se regirá por normas técnicas vigentes para este tema. Sin embargo, dichas normas no existen en el país y en tal sentido se ha entregado una propuesta en la Dirección de Control Sanitario del MSP, de Normas de Control de calidad para fitofármacos: esperamos que muy pronto apruebe definitivamente el Ministro de Salud y entonces podamos trabajar con un respaldo legal completo, para obtener y comercializar productos fitofarmacéuticos de calidad.

El proceso de elaboración de esta propuesta de normas técnicas para el control de la calidad de los fitoterápicos, se comenzó con el auspicio de la OMS-OPS-Proyecto de Holanda, trabajo que no 
se culminó por falta de financiamiento. Por ello la Asociación de Naturistas del Ecuador (ASONATURA) propició una asesoría, con el auspicio de PROMERCADOS (Proyecto de Cooperación Técnica de la GTZ, Asesoría Integral para el Sector Privado, que financia el Gobierno de Alemania) para la realización de los Proyectos de Normas, que a continuación comentamos:

\section{Proyecto A1 de Norma Ecuatoriana (Obligatoria) "Fitoterá- picos: Droga Cruda. Especificaciones Generales".}

Esta norma establece el procedimiento de muestreo para el control de la calidad de la droga de origen vegetal a emplearse en la elaboración de fitoterápicos así como los requisitos para el envase, etiquetado, embalaje, marcación, transporte y almacenamiento de dicha droga cruda.

Esta norma se aplica a todo producto que se elabora a partir de plantas medicinales destinado al consumo humano con un fin terapéutico. En ella se dispone que los requisitos de calidad particulares de drogas crudas se establecerán en normas particulares acordes a esta norma general tanto como si la droga se encuentra mezclada con plantas tóxicas, insectos, excretas de roedores u otra contaminación se considera no apta para su uso. Se establecen también las condiciones de entrega y todas las especificaciones para el envase, etiquetado, embalaje y marcación, así como todo lo relacionado con la inspección, transporte, manipulación, almacenamiento y conservación de las drogas crudas.

\section{Proyecto A1 de Norma Ecuatoriana (Obligatoria) “Fitoterápicos: Droga Cruda. Métodos de ensayo".}

Esta norma describe los métodos de ensayo para el control de calidad de los requisitos de la droga cruda de origen vegetal que se empleará en la elaboración de los fitoterápicos.

Teniendo en cuenta lo establecido en la literatura internacional (4) (5) (6) la norma establece los métodos de ensayo y su orden como sigue: 
1. Comprobación de requisitos macromorfológicos.

2. Determinación de los requisitos micromorfológicos.

3. Determinación de hojas ennegrecidas.

4. Determinación de flores oscurecidas.

5. Determinación de otras partes de la propia planta.

6. Materia orgánica extraña.

7. Materia inorgánica extraña.

8. Cenizas totales.

9. Cenizas solubles en agua.

10. Cenizas insolubles en ácido clorhídrico.

11. Contenido de humedad.

12. Determinación de sustancias solubles.

13. Determinación de aceites esenciales.

14. Determinación de metales pesados (cadmio y plomo).

15. Determinación de arsénico.

16. Residuos de pesticidas.

17. Determinación de microorganismos.

\section{Proyecto A1 de Norma Ecuatoriana (Obligatoria)} "Fitoterápicos: Extractos vegetales. Métodos de ensayo".

Esta norma, por su parte, describe los métodos de ensayo para el control de calidad de los requisitos de los extractos vegetales que se emplean en la elaboración de fitoterápicos.

Para elaboración de la norma también se tuvo en cuenta la literatura especializada internacional (7) (8), en la que se detallan los siguientes métodos en ese orden:

\section{PARA EXTRACTOS FLUIDOS Y TINTURAS:}

1.1. Descripción organoléptica.

1.2. Determinación del $\mathrm{pH}$.

1.3. Determinación del índice de refracción.

1.4. Determinación de la densidad relativa.

1.5. Determinación de sólidos totales.

1.6. Análisis capilar.

1.7. Contenido alcohólico.

1.8. Identificación del (los) principio(s) activo(s).

1.9. Cuantificación del (los) principio(s) activo(s). 


\section{PARA EXTRACTOS BLANDOS:}

2.1. Descripción organoléptica.

2.2. Solubilidad.

2.3. Determinación del pH.

2.4. Determinación de cenizas.

2.5. Determinación de humedad.

2.6. Determinación de sustancias solubles.

2.7. Identificación del (los) principio(s) activo(s).

2.8. Cuantificación del (los) principio(s) activo(s).

\section{PARA EXTRACTO SECO:}

3.1. Descripción organoléptica.

3.2. Determinación de cenizas.

3.3. Determinación de humedad.

3.4. Determinación de sustancias solubles.

3.5. Identificación del (los) principio(s) activo(s).

3.6. Cuantificación del (los) principio(s) activo(s).

4. Proyecto A1 de Guía Ecuatoriana de Práctica "Guía Práctica para la evaluación y control de la calidad de los fitoterápicos".

El contenido de esta guía se elaboró sobre la base de las pautas para la evaluación de medicamentos herbarios de la OMS (2) y lo reproducimos íntegramente a continuación, por su importancia.

\section{GUÍA DE PRÁCTICA PARA LA EVALUACIÓN Y EL CONTROL DE LA CALIDAD DE LOS FITOTERÁPICOS}

\section{OBJETO}

Esta guía establece las pautas para la evaluación y el control de la calidad de los fitoterápicos, con vistas a la obtención del Registro Sanitario para su comercialización. 


\section{DEFINICIONES}

Para los efectos de esta norma se adoptan las definiciones contempladas en las Normas INEN para Fitoterápicos.

\section{DISPOSICIONES GENERALES}

3.1. En la elaboración de los fitoterápicos se acepta como componentes activos a las drogas crudas o combinaciones de estas, en estado bruto o en forma de extractos vegetales. También se acepta la adición de cualquier sustancia auxiliar o excipiente que permita el acabado de la forma farmacéutica específica: estas sustancias auxiliares son por supuesto farmacológicamente inactivas.

3.2. No son fitoterápicos los componentes que contienen sustancias activas, químicamente definidas o constituyentes de plantas, aislados y/o purificados.

\section{ETAPAS PARA LA INVESTIGACIÓN Y OBTENCIÓN DE UN FITOTERÁPICO}

\section{Selección de la especie vegetal a investigar:}

Conociendo el uso tradicional (información etnofarmacológica), información bibliográfica, resultados de investigaciones en curso, problemas de salud que puede resolver y factibilidad de cultivo de una planta medicinal, se debe realizar su selección para acometer un estudio a fin de obtener un fitofármaco a partir de esta especie vegetal.

\section{Identificación botánica de la especie:}

Para iniciar la investigación, es esencial la precisa identificación botánica de la especie vegetal a partir de las fuentes de suministro para el estudio. 


\section{Caracterización fitoquímica preliminar:}

Antes de realizar la comprobación o validación del efecto farmacológico esperado en la especie vegetal, es necesario realizar un tamizaje fitoquímico preliminar para identificar de manera cualitativa los principales componentes de la planta y determinar la existencia o no de los componentes potencialmente tóxicos.

Esta etapa se complementa con una extensa revisión bibliográfica que determina su extensión al tiempo que proporciona pautas para los estudios farmacológicos y toxicológicos

\section{Estudio farmacológico I:}

En este estudio se busca corroborar o identificar una acción farmacológica supuesta en la planta. En él se deben emplear modelos experimentales que sean imprescindibles y adecuadamente validados, trabajar con animales sanos, procedentes de bioterios y adecuadamente estandarizados, emplear controles positivos y aplicar las buenas prácticas de laboratorio, así como los principios éticos para la investigación en animales.

\section{Estudio toxicológico I:}

Su objetivo es evaluar preliminarmente posibles toxicidades (aguda y genotóxica) del extracto de la planta con actividad farmacológica demostrada. En esta estudio se deben emplear modelos experimentales que sean imprescindibles y adecuadamente validados; controles positivos; el extracto que mostró previamente actividad farmacológica; dosis que difieran notable y considerablemente más superiores y con respecto a las que pudieran exponerse al hombre. También se debe trabajar con animales sanos y que cumplan con los mismos requisitos de la etapa anterior; se tiene que observar el cumplimiento de las normas éticas y las buenas prácticas de laboratorio y emplear cepas de microorganismos reconocidas internacionalmente para la evaluación genotóxica. 


\section{Elaboración y estandarización del fitoterápico:}

En esta etapa se prepara la forma farmacéutica más adecuada atendiendo a las indicaciones y a la vía de administración. También se realizan los estudios para la estandarización de esa forma farmacéutica.

Estandarización de la forma farmacéutica:

Todo el proceso de elaboración se debe estandarizar teniendo en cuenta los siguientes aspectos:

- Especificaciones de calidad de la materia prima y métodos analíticos para su comprobación, que incluya informe de análisis químico y microbiológico de los componentes activos. Si existiera una monografía en la Farmacopea, bastará con aludir a ella, de lo contrario se elaborará la monografía correspondiente.

- víndices de calidad para el producto en proceso y del producto terminado, que señale sus tolerancias y métodos analíticos para su comprobación y adjuntar informe de análisis. De no ser posible la cuantificación del principio activo (lo cual debe quedar debidamente justificado) bastará con identificar una sustancia o mezcla de sustancias características con vista a velar por la calidad uniforme en la preparación.

- Descripción de la tecnología de elaboración y envase del fitoterápico que se complementa con la información de todos los procesos de elaboración y envase que sufre la droga cruda o el extracto vegetal (en el caso que se parta de un extracto para la obtención del producto final).

- Estudio de estabilidad del producto terminado, que se completará con la información que derive de la droga cruda o extracto vegetal empleados para su preparación. Establecer fecha de vencimiento del producto. En este estudio de estabilidad hay que tener en cuenta los siguientes aspectos: 
* Los estudios deberán realizarse en el material de envase propuesto para su comercialización.

* Las condiciones del estudio de estabilidad serán: $30^{\circ} \mathrm{C}$ y $70 \%$ de humedad relativa.

* Evaluar el efecto de la luz.

* La frecuencia de los análisis será trimestral para las propiedades físicas; semestral para las químicas y anual en el caso que se requiera evaluación biológica.

* En el caso de la evaluación química si no es posible evaluar el principio activo, utilizar un perfil cromatográfico.

* En el caso de que no haya elementos suficientes o las propiedades farmacológicas o toxicológicas de la sustancia activa o de los productos de degradación lo requiera; se deberá comprobar la estabilidad del producto terminado con una evaluación biológica (microbiológica, farmacológica o toxicológica) en el tiempo y comparada con la inicial.

\section{Agrobiología:}

El objetivo de esta etapa es realizar los estudios necesarios que permitan garantizar el suministro del material vegetal en cantidades suficientes para la comercialización, con las mismas condiciones o calidad.

Este estudio debe considerar por lo menos los siguientes aspectos:

- Preparación del suelo.

- Método de plantación.

- Calendario de siembra.

- Norma de semilla.

- Ciclo vegetativo.

- Rendimiento agrícola.

- Cosecha. 
- Atenciones culturales.

- Fertilizantes.

- Riego.

- Parte útil de la planta.

- Procesos post-cosecha: desinfección, secado, envase y almacenamiento.

\section{Farmacología II:}

Esta fase complementa los estudios farmacológicos iniciados en la Farmacología I, que evalúan la farmacodinámica y de ser posible su farmacocinética (cuando se conoce el principio activo). Estos estudios deben incluir los siguientes aspectos:

- Dosis efectiva.

- Potencia o actividad relativa.

- Índice terapéutico.

- Mecanismo de acción (cuando sea posible).

- Duración de la acción.

\section{Toxicología II:}

Esta fase complementa los estudios iniciados en la Toxicología I, debe incluir los siguientes aspectos:

- Toxicidad subcrónica.

- Genotoxicidad.

\section{Ensayo clínico:}

Estos estudios deben evaluar inequívocamente la actividad terapéutica y efectos secundarios, así como reacciones adversas en el humano. Para ello se procederá según las regulaciones vigentes en el país, para estos estudios.

\section{Registro y comercialización:}

Se procede según lo establecido en Reglamento de productos fitoterápicos del Ecuador. 
De acuerdo a las diferentes categorías de fitoterápicos que establece dicho Reglamento se cumplirán o no, todas las etapas antes mencionadas, según corresponda.

\section{ETAPA DE PRODUCCIÓN PARA LA COMERCIALIZACIÓN}

\section{Estandarización de la materia prima (droga cruda):}

Indicaciones para los proveedores de droga cruda:

Los productores-proveedores de droga cruda vegetal deben garantizar los Estudios Agrotécnicos necesarios descritos en 4.7.

$\underline{\text { Indicaciones al productor de fitoterápicos: }}$

Siempre que la droga cruda no aparezca en la Farmacopea oficial del país, los productores deben elaborar para cada droga cruda las Normas de Control de la Calidad específicas, sobre la base de las Normas INEN generales. Para esto es necesario que los parámetros o requisitos de calidad establecidos para las drogas crudas sean estandarizados, para lo cual se debe trabajar con material vegetal colectados al menos en tres localidades diferentes del país y en tres épocas distintas del año. En estas condiciones se pueden establecer rangos mínimos y máximos para cada parámetro de calidad y proponer la norma de requisitos correspondiente a la autoridad competente para su aprobación.

\section{Comprobación de los requisitos de calidad de la droga cruda o extracto vegetal:}

El productor tiene la obligación de comprobar los requisitos de calidad establecidos en las normas específicas para cada droga o extracto vegetal. En el caso de que la droga o el extracto no tenga la norma específica elaborada, entonces determinara los requisitos de calidad según Normas INEN.

Una vez que se ha determinado si la droga cruda o el extracto vegetal cumple con los requisitos, comienza el proceso de producción del fitoterápico. 


\section{Controles en proceso y del producto terminado:}

Se realizan según lo establecido para cada forma farmacéutica en particular en la Farmacopea oficial en el país.

\section{CONCLUSIONES}

En el Ecuador el uso de Plantas Medicinales en forma de productos farmacéuticos acabados y presentados en formas farmacéuticas conocidas, ha proliferado considerablemente. Sin embargo, no en todos los casos se cumplen normas técnicas para la elaboración de esos productos con calidad, ni se ha validado la actividad terapéutica que se declara en las indicaciones de las etiquetas. Por todo ello que se hace necesario la puesta en marcha de la reglamentación que se ha mencionado, así como el control sobre la base de la propuesta de normas técnicas. De esta manera se puede comenzar a regular la producción y comercialización de estos productos y sobre todo lograr equiparar la Fitoterapia con las otras terapias que se utilizan en el país, para lo cual se hace necesario, realizar una serie de actividades (9)

- Establecer Herbarios institucionales equipados.

- Jardines botánicos educativos.

- Contar con taxónomos con experiencias y especializados.

- Conservación de germoplasma nativo.

- Manejo agrotecnológico de bosques.

- Cultivo orgánico de plantas medicinales.

- Estudios de rentabilidad económica.

- Validar las actividades biológicas in vitro, in vivo y clínicamente.

- Tecnificar los procesos de la industria fitofarmacéutica.

- Estandarizar los procedimientos y normalizar las materias y productos.

- Modernización de la industria.

- Incorporación al mercado farmacéutico 
- Prescripción por los sectores médicos.

- Apertura de mercados estratégicos.

- Incorporar a los Pensum de estudios el tema de fitofármacos.

- Realización de Seminarios a nivel nacional.

- Capacitación de promotores.

- Información específica para todos los niveles.

- Participación de todos los sectores involucrado en todo el proceso.

Una vez cumplimentadas todas esas actividades y/o necesidades podremos hacer realidad la dialéctica del desarrollo fitoterapéutico (9)

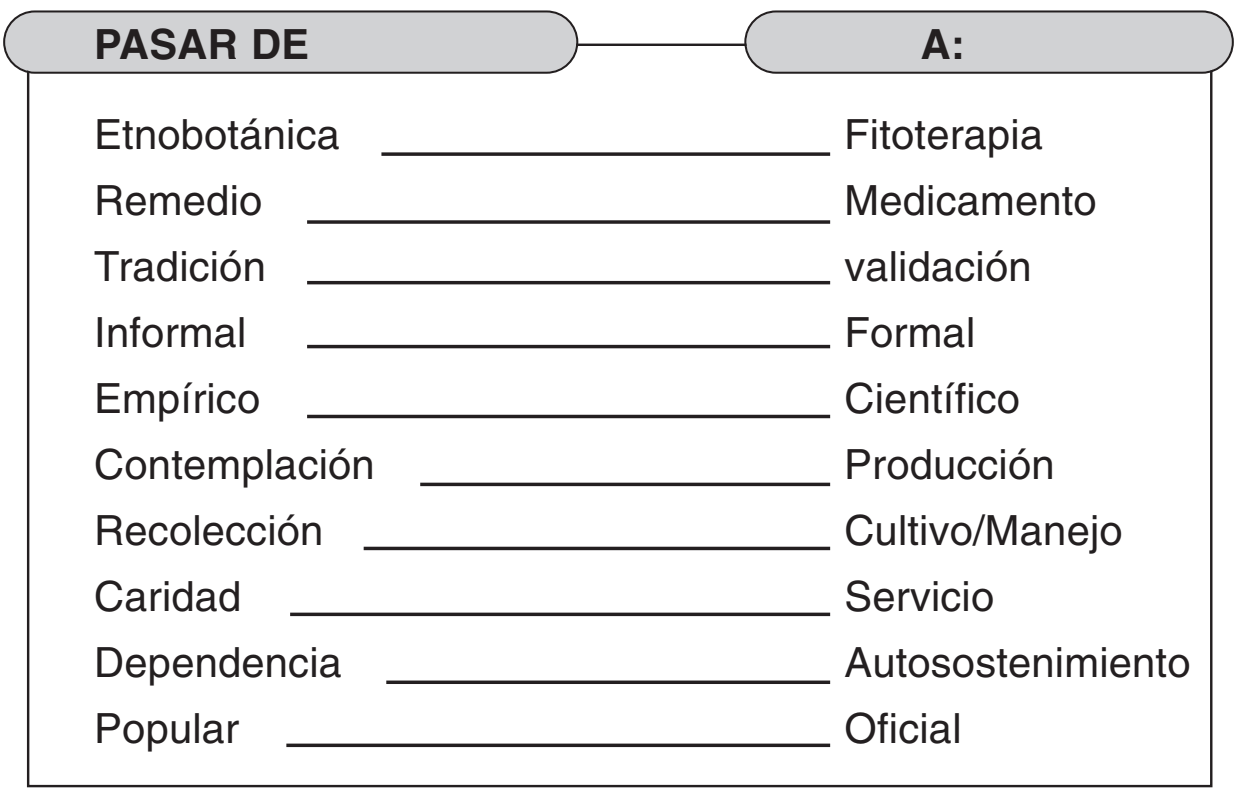

Solo queda exhortar y exigir a las autoridades competentes del país a que continúen, de la manera más inmediata, con el trámite de rigor correspondiente, que permita hacer realidad algo que es indispensable para todos los interesados en esta Alternativa Terapéutica: ellos son los que producen y comercializan fitoterápicos y sobre todo la población que recibe este servicio como una alternativa económica y que tiene que ser de calidad. 


\section{REFERENCIAS BIBLIOGRÁFICAS}

1. Montes, G. M. "Fitoterapia y gerontoprofilaxis". En: Homeopatía y Naturaleza. Rev. De Difusión Homeopática. Editada por Fundación R. Knop. Año 3. Número 3. 1993. pp 21-30.

2. O.M.S. Pautas para la Evaluación de Medicamentos Herbarios. Programa de Medicina Tradicional. Ginebra. 1991.

3. Dehesa M.A. Informe de consultoría. PROMERCADOS. Julio, 1996.

4. World Health Organization. Quality Control Methods for Medicinal Plant Materials. Switzerland, July 1992.

5. Norma Cubana NRSP 309. Medicamentos de origen vegetal: Droga Cruda. Métodos de ensayo. MINSAP. La Habana, Cuba, 1991.

6. Norma Cubana NRSP 310. Medicamentos de origen vegetal: Droga Cruda. Especificaciones generales. Ministerio de Salud Pública (MINSAP) La Habana, Cuba 1991.

7. Norma Cubana NRSP 311. Medicamentos de origen vegetal: Extractos y Tinturas. Procesos Tecnológicos. MINSAP. La Habana, Cuba. 1991.

8. Norma Cubana NRSP 312. Medicamentos de origen vegetal: Extractos y Tinturas. Métodos de Ensayo. MINSAP. La Habana, Cuba. 1991.

9. Cáceres A. Curso de Química y Tecnología de Fitomedicamentos. Universidad Federal Fluminense, Niteroi, Brasil. 1997. 